\title{
A ERMANNO CALDERA IN MEMORIAM
}

La admiración y el afecto que Ermanno Caldera ha suscitado entre colegas y discípulos, en todo lugar y en todo tiempo, han motivado este merecido homenaje. La satisfacción que nos causa el poder ofrecer dicha monografía dedicada al Romanticismo Español e Hispanoamericano es manifiesta, pues gracias a su publicación podemos expresar nuestro agradecimiento y el reconocimiento público de quienes hemos sido partícipes de su amistad y su sabiduría.

Por estas fechas le hubiese correspondido ser investido Doctor Honoris Causa por la Universidad de Alicante, honor merecido por su ejemplar dedicación al enriquecimiento del hispanismo.

ENRIQUE RUBIO CREMADES 



\section{ERMANNO CALDERA}

\section{Curriculum vitae}

Datos Personales

Lugar y fecha de nacimiento: Turín, 16-07-1923.

1948: Licenciado en Letras (Universidad de Turín).

1950-1973: Profesor y luego director de Instituto.

1965: 'Libero docente' en Lengua y Literatura española.

1966-1975: Encargado de Lengua y Literatura Española en la Universidad de Génova.

1970-1974: Encargado de Lengua y Literatura Española en la Universidad de Turín.

1975-1993: Catedrático de Lengua y Literatura Española en la Universidad de Génova.1993-1995: Emérito («fuori ruolo»).

Desde 1995: Jubilado.

2001: Inauguración del Portal de Hispanistas Ermanno Caldera en la Biblioteca Virtual Miguel de Cervantes de la Universidad de Alicante.

2003: La Junta de Facultad de Filosofía y Letras de la Universidad de Alicante aprobó por unanimidad la propuesta del Departamento de Filología Española, Lingüística General y Teoría de la Literatura el nombramiento de Doctor Honoris Causa por la Universidad de Alicante.

Fallecimiento: 19 de febrero de 2004.

2005: Donación de su biblioteca a la Universidad de Alicante.

\section{Datos Académicos}

Ex director del departamento de Lenguas y Literaturas Extranjeras en la Facultad de Magisterio de la Universidad de Génova.

Ex presidente del Curso de Licenciatura en Lenguas y Literaturas Extranjeras de la misma Facultad.

Ex presidente de la Asociación de los Hispanistas Italianos (AISPI). 
Ex presidente del «Centro Internacional de Estudios sobre el Romanticismo Hispánico».

Conferencias, clases y seminarios en varias universidades y centros culturales de España, Italia, Francia, Alemania, Israel, Estados Unidos, Canada.

Ha organizado siete Congresos sobre el romanticismo hispánico.

Ha organizado el Congreso sobre teatro de magia y santos en Valladolid.

Ha editado volúmenes misceláneos y ha dirigido la colección «Tramoya» de teatro.

\section{Bibliografía}

Libros

Il teatro di Moreto, Pisa, Goliardica, 1960, $236 \mathrm{pp}$.

Primi manifesti del romanticismo spagnolo, Pisa, Istituto di Letteratura Spagnola, 1962, $126 \mathrm{pp}$.

Il dramma romantico in Spagna, Pisa, Università, 1974, 235 pp.

La commedia romantica in Spagna, Pisa, Giardini, 1978, 194 pp.

El teatro español en la época romántica, Madrid, Castalia, 2001

\section{Artículos}

«Un motivo delle commedie 'de enredo': l'elaborazione de El Melancólico», Studi Tirsiani, Milano, Feltrinelli, 1958, pp. 93-100.

«Solitudine dei personaggi di Rojas», Studi Ispanici, Milano, Feltrinelli, 1962, pp. 37-60.

«Il problema del vero nelle Escenas Matritenses», Miscellanea di Studi Ispanici

Pisa, Istituto di Letteratura Spagnola, 1964, pp. 101-121.

«L'oratoria nel Poema de Mio Cid», Miscellanea di Studi Ispanici, Pisa, Istituto di

Letteratura Spagnola, 1965, pp. 5-29.

«En torno a las tres primeras estrofas del Polifemo de Góngora», Actas del Segundo

Congreso Internacional de Hispanistas, Nimega, 1967, pp. 227-233. «Retorica, narrativa e didattica nel Conde Lucanor», Miscellanea di Studi Ispanici,

Pisa, Istituto di Letteratura Spagnola, 1967, pp. 5-120.

«Il tema dell'ausencia in Garcilaso», Studi di Letteratura Ibero-Americana, 2,

Milano-Varese, Istituto Editoriale Cisalpino, 1968, pp. 1-16.

«L'estetica di Garcilaso», Miscellanea di Studi Ispanici, Pisa, Istituto di Letteratura

Spagnola, 1968, pp. 181-207.

«Ancora sulla viola di Garcilaso», Studi di Letteratura Spagnola, Roma, 1968-70, pp. 267-270.

«EI manierismo en San Juan de la Cruz», Prohemio, I, 3 (1970), pp. 333-355. 
«Per uno studio delle varianti nelle Rimas di Bécquer», Quaderni Iberoamericani, 39-40 (1971), Torino, pp. 225-233.

«I simboli del fuoco e dell'acqua nel Burlador de Sevilla», Il Superuomo, Firenze,

La Nuova Italia, 1971, pp. 41-58.

«Rintocchi di campane nella letteratura romantica», Il Superuomo, II, Firenze, La

Nuova Italia, 1972, pp. 103-117.

«La condanna del superuomo in Lope de Vega», Il Superuomo, III, Firenze, La

Nuova Italia, 1973, pp. 53-55.

«I satanici del romanticismo spagnolo», Il Superuomo, V, Firenze, La Nuova Italia, 1976, pp. 173-190.

«Il metateatro di Ramón de la Cruz», Linguistica e Letteratura, II, 2 (1977), Pisa,

Giardini, pp. 81-113.

«ll riformismo illuminato nei sainetes di Ramón de la Cruz», Letterature 1 (1978),

Genova, pp. 31-50.

«P. Napoli Signorelli traduttore di Moratín», Studi di Letteratura italiana in onore di F. Montanari, Genova, Il Melangolo, 1980, pp. 149-160.

«Da Menschenhass und Reue a Misantropía y arrepentimiento: storia di una traduzione», Studi Ispanici, Pisa, Giardini, 1980, pp. 187-209.

«Per una definizione di 'simbolo'», Momenti di simbolismo figurativo, Saluzzo,

Museo Civico Casa Cavassa, 1980, pp. 1-4.

«L'influenza di Shakespeare sul romanticismo spagnolo (a proposito di Romeo y

Julieta di Solís-Ducis)», Letterature 3 (1981), Genova, pp. 41-56.

«Le iperboli di González del Castillo», Aspetti e problemi delle letterature iberi-

che. Studi offerti a F. Meregalli, Roma, Bulzoni, 1981, pp. 79-93.

«La última etapa de las comedias de magia», Actas del Séptimo Congreso de la AIH, Roma, Bulzoni, 1982, pp. 247-253.

«De Aliatar a Don Álvaro: sobre el aprendizaje clasicista del Duque de Rivas»,

1) Romanticismo 1, Genova, Biblioteca di Letterature, 1982.

2) Cuadernos de filología, III, 3 (1983), Valencia, pp. 5-31.

«Misticismo e tramoya: Santa Teresa in alcune comedias de santos», Letterature,

5 (1982), Genova, pp. 89-108.

«Il teatro romantico in Spagna», Problemi del romanticismo, Milano, Shakespeare

\& Company, 1983, pp. 322-346.

«Sulla spettacolarità delle commedie di magia», Teatro di magia, a cura di E.

Caldera, Roma, Bulzoni, 1983, pp. 11-32.

«La magia nel teatro romantico», ibidem, pp. 185-205.

«La polisemia delle porte in Historia de una escalera», Letterature, 6 (1983),

Genova, pp. 193-204.

«Calderón desfigurado (Sobre las representaciones calderonianas en la época prerromántica)», Anales de Literatura Española, II (1983), Alicante, pp. 5781 . 
«ll sogno è vita e la vita non è commedia nelle piezas cortas di Calderón», Colloquium calderonianum internationale, Atti, L'Aquila, UniversitàInstituto Español de Cultura-Teatro Stabile, 1983, pp. 289-295.

«Sulla fortuna del Pellico in Spagna», Saluzzo e Silvio Pellico, Torino, Centro di

Studi Piemontesi, 1984, pp. 75-76.

«L'età della ragione», Teatro romantico spagnolo, Bologna, Patron, 1984, pp. 7-

22.

«La Pata de cabra e Le pied de mouton», Studia historica et philologica in hono-

rem $M$. Batllori, Roma, Instituto Español de Cultura, 1984, pp. 567-575.

«Sobre el tema de este Congreso», Romanticismo 2, Genova, Biblioteca di

Letterature, 1984, pp. 9-13.

«Il dramma esemplare di Pedro Crespo, uomo 'discreto'», P. Calderón de la Barca,

L'Alcalde di Zalamea, Genova, Teatro di Genova, 1985, pp. 30-44.

«L'Inquisizione e il fanatismo religioso nel teatro spagnolo del primo Ottocento»,

Letterature 8 (1985), Genova, pp. 27-42.

«Entre cuadro y tramoya», Dieciocho, 9, 1-2 (1986), Rotgers University-Cornell

University, pp. $51-56$.

«Hispanismo italiano, 1808-1868», Árbor, CXXIV (1986), pp. 95-99.

«Poetizar la verdad en Fernán Caballero», Romanticismo 3-4, Genova, Biblioteca

di Letterature, 1988, pp. 17-22.

«Teatro di magia e secolo dei lumi», Letterature, 11 (1988), Genova, pp. 51-59.

«El teatro en el siglo XIX (I: 1808-1844)», Historia del teatro en España, II,

Madrid, Taurus, 1988, pp. 377-565-610-621.

«De la comedia de santos barroca a la comedia de magia dieciochesca: El mágico lusitano», Coloquio Internacional sobre el teatro español del siglo XVIII, Abano Terme, Piovan, 1988, pp. 99-111.

«Echegaray tra la parola e il silenzio», Symbolae Pisanae, Pisa, Giardini, 1989, pp. 85-98.

«La magia negada: El hechizo sin hechizo de Salazar y Torres», Diálogos hispánicos de Amsterdam, 8/II, Amsterdam-Atlanta, Rodopi, 1989, pp. 311-322. «Las jitanjáforas del Teatro fantástico y anti-hipocóndrico de Monsieur Débout», La Chispa 89, New Orleans, Tulane University, 1989, pp. 53-62.

«La perspectiva femenina en el teatro de Joaquina Balmaseda y Enriqueta Lozano»,

Escritoras románticas españolas, Madrid, Fundación Banco Exterior, 1990, pp. 207-216.

«La letteratura di riflessione ed erudizione» (pp. 747-753); «La narrativa in verso e prosa»; «La lirica»; «Il teatro» (pp. 758-771), Storia della civiltà letteraria spagnola, Torino, UTET, 1990, cap. XI («Dal 1808 al 1869»).

«Bretón o la negación del modelo», Cuadernos de teatro clásico, 5 (1990), Madrid, pp. 141-153. 
«Sobre algunos aspectos del romanticismo español, Toledo romántico, Toledo, Colegio Universitario, 1990, pp. 11-17.

«Il Paso Honroso di Rivas fra storia e leggenda», Dialogo, Studi in onore di L Terracini, Roma, Bulzoni, 1990, pp. 99-116.

«Sulle comedias de santos di Salvo y Vela e sulle fonti del Mágico de Salerno», Letterature, 13 (1990), Genova, pp. 53-60.

«Il teatro del pathos e dell' orrore», EntreSiglos, 1, Roma, Bulzoni, 1991, pp. 57-74.

«1 teatro fantastico», Teatro di magia 2, a cura di E. Caldera, Roma, Bulzoni, 1991, pp. 7-32.

«Un teatro-verità», Teatro politico spagnolo del primo Ottocento, a cura di E.

Caldera, Roma, Bulzoni, 1991, pp. 7-17.

«La passione ispanica e l' eredità romantica in Arturo Farinelli», T. Heydenreich

(ed.), Cultura italiana e spagnola a confronto: anni 1918-1939, Tübingen, Stauffenburg, 1992, pp. 13-20.

«Rimitizzazione e demitizzazione di Cristoforo Colombo», Letterature 14 (1992),

Genova, pp. 104-121.

«La fórmula de Salvo y Vela», La comedia de magia y de santos, Madrid, Júcar, 1992, pp. 321-339.

«Horror y Pathos en los dramones de principios del siglo XIX», Actas del Décimo

Congreso de la AIH, Barcelona, PPU, 1992, II, pp. 1221-1228.

«De la tragedia neoclásica al drama histórico romántico: por qué y cómo», EntreSiglos 2, Roma, Bulzoni, 1993, pp. 67-74.

«Presenza del teatro sentimentale nella Conjuración de Venecia», El Girador,

Studi... offerti a G. Bellini, Roma, Bulzoni, 1993, pp.109-115.

«El tiempo de Don Juan», Ínsula, 564 (1993), Madrid, pp. 14-15.

«Un muestrario romántico (La segunda parte del Zapatero y el Rey)», Lazarillo, 5

(1994), Salamanca, pp. 29-34.

Il teatro barocco nella prospettiva letteraria del primo Ottocento», Spagna

Contemporanea, 5 (1994), Torino, pp. 11-18.

«Liberalismo y anticarlismo en la dramaturgia romántica», Crítica Hispánica,

Duquesne University, Pittsburgh, XVI (1994), n. ${ }^{\circ} 1$, pp. 103-117.

«Clásicos y Románticos frente al teatro del Siglo de Oro», Spanien in der

Romantik, Köln-Weimar-Wien, Böhlau Verlag, 1994, pp. 267-274.

«La sonrisa romántica», Romanticismo 5, Roma, Bulzoni, 1995, pp. 43-49.

«L'antiromanticismo di Ventura de la Vega», Saggi in onore di Giovanni Allegra,

Perugia, Università, 1995, pp. 41-50.

«La polémica sobre el Don Álvaro», Crítica Hispánica, Duquesne University, Pittsburgh, XVII (Spring 1995), pp. 22-35.

«La società spagnola fra il 1810 e il 1820 vista dal palcoscenico», Spagna Contemporanea, Torino, IV (1995), nº 7, pp. 131-139. 
«De lo que hoy se llama romanticismo», Siglo diecinueve, Valladolid, 1 (1995), pp. 77-90.

«Vero e verosimile dal teatro neoclassico al teatro romantico», La festa teatrale ispanica, Napoli, Istituto Universitario Orientale, 1995, pp. 345-353.

«La comedia romántica o del casticismo», Historia y crítica del teatro de comedias del siglo XIX... Y la burguesía también se divierte, El Puerto de Santa María, Fund. P. Muñoz Seca, 1995, pp. 13-26.

«Sobre los ideales neoclásicos y su realización escénica», Teatro Español del siglo XVIII, Lleida, Universidad, 1996, I, pp. 169-180.

«El amor y el tiempo en el Don Juan Tenorio», Actas del Congreso sobre José Zorrilla, Una nueva lectura (18-21 Oct.1993), Valladolid, UniversidadFund. Jorge Guillén, 1995, pp. 13-23.

«Il postgongorismo di Lorca: dalla 'desrealización' all' 'antirealtà' », Letterature,

18 (1995), pp. 113-119.

«Il Martin Fèr», Studi Piemontesi, XXV, 1 (1996), pp. 127-132.

«Presenza italiana nel romanticismo spagnolo», L'Italie dans l'Europe romantique, Moncalieri, CIRVI, 1996, I, pp. 327-337.

«Macarronini I: una satira contro Amedeo d'Aosta», Scrittori 'contro': modelli in discussione nelle letterature iberiche, Atti del Congresso AISPI di Roma, 15-16 marzo 1995, Roma, Bulzoni, 1996, pp. 121-128.

«Sobre las tragedias clasicistas de los románticos», Salina, Tarragona, 10 (1996), pp. 127-134.

«La vocación costumbrista de los románticos», Romanticismo 6, Roma, Bulzoni, 1996, pp. 45-52.

«Ciudad y campo en el teatro bretoniano», Teatro y ciudad, $V$ Jornadas de teatro, Burgos, Universidad, 1996, pp. 35-52.

«Su possibili fonti italiane delle Serranillas», Serena ogni montagna, Studi di Ispanisti Amici offerti a Beppe Tavani, Roma, Bulzoni, 1997, pp. 33-42.

«Sulla ricezione ottocentesca del teatro del Siglo de Oro», Letterature, 19 (1996), pp. 14-21.

«El Quijote en el teatro español del siglo XIX», Actas del Simposio Internacional'97

Nanjing «Cervantes en el mundo», Nanjing, Press Yilin, China, 1997, pp. 17-22.

«Historia de mi corazón. Soggettivismo romantico nei Recuerdos americani di Zorrilla», Un lume nella notte. Studi... a Giuseppe Bellini, Roma, Bulzoni, 1997, pp. 33-40.

«Literatura española del siglo XIX», Los estudios hispánicos en Italia y en Alemania, Actas del congreso Villa Vigoni 21-23 de mayo de 1989, Bochum,

Romanisches Seminar, Ruhr Universität, 1993, pp. 81-84.

«Antes y después del Tenorio», Studies in Honor of Gilberto Paolini. Newark, Cuesta, 1996, pp. 159-169. 
«El costumbrismo ideológico y literario de La Gaviota», Costumbrismo andaluz. (Eds. Álvarez Barrientos-Romero Ferrer), Sevilla, Universidad, Secretariado de Publicaciones, 1998, pp. 33-46.

«Del teatro romántico a la alta comedia», Actas del Coloquio «Del romanticismo al realismo», Barcelona, Publicacions Universitat de Barcelona, 1998, pp. 141-146.

«Il viaggio aereo di Simplicio Bobadilla Majaderano y Cabeza de Buey», Il viaggio e le letterature ispaniche, Atti della Tavola Rotonda, Napoli, 12 e 13 dic. 1996, Napoli, L'Orientale, 1998, pp. 91-94.

«Mario Damonte» [necrologio], Atti dell'Accademia Ligure di Scienze e Lettere, Serie V, LIV, 1997, pp. 56-58.

«Sobre las fuentes del Don Juan Tenorio de Zorrilla», Convegno di Studi Ispanici in memoria di Mario Damonte, Genova, Accademia Ligure di Scienze e Lettere, 1998, pp. 125-140.

«Il matrimonio sulle scene settecentesche e ottocentesche», Signoria di parole, Studi offerti a Mario Di Pinto, Napoli, Liguori, 1998, pp. 111-123.

«Diferentes maneras de traducir a Scribe», La traducción en España (1750-1830).

Lengua, Literatura, Cultura (Ed. F. Lafarga), Edicions de la Universitat de Lleida, 1999, pp. 429-436.

«El actor en las reseñas periodísticas del teatro romántico español», Dramaturgies romantiques, textes réunis par G. Zaragoza, EUD (Editions Universitaires de Dijon), 1999, pp. 133-143.

«Árabes y judíos en la perspectiva cristiana de Juan Manuel», Salina, XIII (1999), pp. $37-40$.

«Espectáculos teatrales. Siglo XIX», Historia de los espectáculos en España (eds.

A. Amorós-J.M. Díez Borque); Madrid, Castalia, 1999, pp. 87-103.

«La poesía romántica vista por los románticos», en Romanticismo 7. La poesía romántica, Piero Menarini (ed.), Bologna, II Capitello del Sole, 2000, pp. 29-36.

«El teatro romántico juzgado por los románticos (Itinerario del canon en el Semanario Pintoresco Español)», Actas del II Coloquio de la Sociedad de Literatura Española del Siglo XIX: La elaboración del canon en la literatura española del Siglo XIX, PPU, 2002, pp. 97-104.

«Los románticos se burlan de sí mismos. Algunos apuntes sobre el Romanticismo existencial», Romanticismo 8. Los románticos teorizan sobre sí mismos, Bologna, II, Capitello del Sole, 2002, pp. 63-75.

«La liberación 'teatral' de las mujeres en las primeras piezas románticas», Actas del III Coloquio de la Sociedad de Literatura Española del Siglo XIX: Lectora, Heroína, Autora (La mujer en la literatura española del siglo XIX), Barcelona, PPU, 2005, pp. 57-63. 
Estudios críticos, reseñas y traducciones

P. Calderón, Teatro, trad. a cura di C. Berra e E. Caldera, Torino, UTET, 1958 (trad. de El Mágico prodigioso, La dama duende).

Reseña en Quaderni Iberoamericani, 27 (1961), Torino, pp. 185-186 (G.A. Bécquer, Del olvido en el ángulo oscuro).

Reseñas en Quaderni Iberoamericani, 29 (1963), Torino, pp. 29l-297 (M.R. Lida, Two Spanish Masterpieces; M. Bataillon, La Célestine; Comedia de Calixto y Melibea, ed. facsimil).

Reseñas en Studi di Lingua e Letteratura Spagnola, Torino, Giappichelli, 1965, pp. 335-340 (Armistead-Silverman, Diez romances hispánicos).

Reseñas en Quaderni Iberoamericani, 34 (1967), Torino, p. 114 (Cinti, Letteratura e Politica in J.A. de Vera).

Reseñas en Quaderni Iberoamericani, 37 (1969), Torino, pp. 50-52 (P. López de Ayala, Las Flores de los 'Morales de Job').

Reseña en Quaderni Iberoamericani, 47-48 (1975-76), pp. 404-405 (D.L. Shaw, The Generation of 1898 in Spain).

Reseña en Rassegna Iberistica 13 (1982), pp. 52-53 (Allegra, La viña y los sur$\cos )$.

Reseña en Rassegna Iberistica 14 (1982), pp. 46-47 (Berichte der diplomatischen Vertreter der Wiener Hofes).

Ramón de la Cruz, Marta Abandonada (Introducción, pp.7-36), Roma, Bulzoni, 1984.

Don Álvaro o La fuerza del sino, Madrid, Taurus, 1986 (Estudio preliminar, pp. 1-74; Texto y notas).

Reseña en Rassegna lberistica 33 (1988), pp. 49-51 (Gies, Theatre and Politics). José Echegaray, O pazzia o santità, Napoli, Liguori, 1994, 247 pp. (Introducción, pp. 1-52. Texto, traducción y notas).

Duque de Rivas, Don Álvaro o la fuerza del sino, Barcelona, Crítica, 1994 (Estudio Preliminar, pp. IX-XXII).

Reseñas en Letterature, 19 (1996), pp. 119-121 (A. Muñoz Sanz, O Yacoi; A. Cancellier, Lenguas en contacto).

Reseña en Letterature 20 (1997), pp. 159-161 (López, Ros de Olano, Sáenz, J. Somoza, Bernard, Solís, El Pastelero de Madrigal).

Reseña en Dieciocho, 22.1 (1999), pp. 155-156 (Cañizares, Juan de Espina). 